\title{
Osseodensification-driven Maxillary Sinus Lift along with Sticky Bone Augmentation: A Novel Concept for Dental Implant Placement
}

\author{
Sumit Munjal ${ }^{1}$, Seema Munjal ${ }^{2}$
}

\begin{abstract}
Aim and objective: The purpose is to present a novel technique for normal sinus lift in the upper jaw that is feasible in cases where other approaches are contraindicated. Acknowledging indigenous growth factors and precise osseodensification (OD) significantly reduces biological costs.

Background: The lowest success of implant rehabilitation in the posterior maxilla has made the surgeons ponder on the frequent reduction of alveolar ridge height beneath the sinus. Although the direct sinus lift and osteotomes addressed the issue, the associated risks of parasthesia, perforation, and patient morbidity were a point of concern.

Case description: A 51-year-old male patient's blood was centrifuged emulating a standard protocol. Densah Burs were used to prepare the fixture site on OD principles. Next, the platelet-rich fibrin (PRF) extracted from the spin-off was mixed with the graft NB Dental Morsels, to form a sticky bone. As planned, the $3.75 \times 10 \mathrm{~mm}$ implant, drove the material under $35 \mathrm{rpm}$ into the final desired osteotomy depth. Upon ensuring a $25-\mathrm{Ncm}$ torque stability, a healing abutment was tightened over it and the flap was sutured finally.

Conclusion: The explicated implant methodology is feasible in a dental setup for its ease and non-invasiveness.

Clinical significance: A relatively new technique demonstrated in our study provides a choice of modality in sinus floor elevation (SFE) in such circumstances.

Keywords: Crestal sinus lift, Osseodensification, Sinus membrane, Sticky bone.

International Journal of Experimental Dental Science (2021): 10.5005/jp-journals-10029-1219
\end{abstract}

\section{BACKGROUND}

The edentulous patient may be unable to recover normal function, esthetics, comfort, or speech with a traditional prosthesis. ${ }^{1}$ Due to the high success rates, the insertion of dental implants has become a knowable treatment solution for several patients. ${ }^{2}$ Highest levels of success rate are reported in the anterior mandible, followed by the posterior mandible and anterior maxilla. The maxillary sinus retains its overall size when teeth remain in function, but the ensuing osteoclastic activity on tooth loss causes both lateral, as well as inferior expansion. Unequivocally, the posterior maxilla has the lowest success of any region. ${ }^{3}$

A noteworthy limitation in the implant realm is pneumatization that occurs at the expense of alveolar ridge height beneath the maxillary sinus. ${ }^{4}$ Also, both the bone quality and quantity are impacted and the compromised bone may cause both early and late failures. ${ }^{5}$ Surgically maneuvering the crestal sinus floor elevation (SFE) can dramatically increase the height of bone available below. This approach can gain approximately $3-5.0 \mathrm{~mm}$ in height within the sinus with a simultaneous implant placement option. ${ }^{6}$

The standard drilling (SD) served to prepare and cut bone for fixture installation so far. Osseodensification (OD) technique is the recent introduction, which utilizes proprietary high-speed densifying burs (Fig. 1) to preserve and compact the bone at the site. ${ }^{7}$ Maintaining and preserving bone during osteotomy preparation leads to increased primary stability, which then enhances implant secondary stability. ${ }^{7,8}$ The present article reports the novel case of indirect sinus lift using OD concept with simultaneous implant placement along with an adjunct platelet-rich fibrin (PRF).
${ }^{1}$ Department of Periodontics, ITS Dental College, Greater Noida, Uttar Pradesh, India

${ }^{2}$ Department of Prosthodontics, ITS Dental College, Greater Noida, Uttar Pradesh, India

Corresponding Author: Sumit Munjal, Department of Periodontics, ITS Dental College, Greater Noida, Uttar Pradesh, India, Phone: +91 9910259040, e-mail: ssmunjal@yahoo.co.in

How to cite this article: Munjal S, Munjal S. Osseodensification-driven Maxillary Sinus Lift along with Sticky Bone Augmentation: A Novel Concept for Dental Implant Placement. Int J Experiment Dent Sci 2021;10(1):45-47.

Source of support: Nil

Conflict of interest: None

\section{Case Description}

A 51-year-old male patient, non-smoker, and non-Bruxer reported to the Faces $n$ Braces Dental Institute, New Delhi with a desire to replace the previously extracted tooth 17 (Fig. 2A) 6 months ago. No history of chronic sinusitis or long-standing nasal obstruction was noted. X-rays and computerized tomographic (CT) scan revealed (Figs 2B and C) an adequate buccolingual cortical bone width. Although the crest to the sinus floor at the edentulous area was $<4 \mathrm{~mm}$ distant (Fig. 2D), yet it was decided to perform the implant installation of length $10 \mathrm{~mm}$.

Upon the written consent, the flap was elevated using midcrestal and crevicular incisions. The osteotomy was started with the pilot drill in a clockwise fashion and then with the Densah ${ }^{\text {TM }}$ Bur (Fig. 2E) at 900 rpm speed. The vertical orientation of the first bur (Fig. 2F) was 

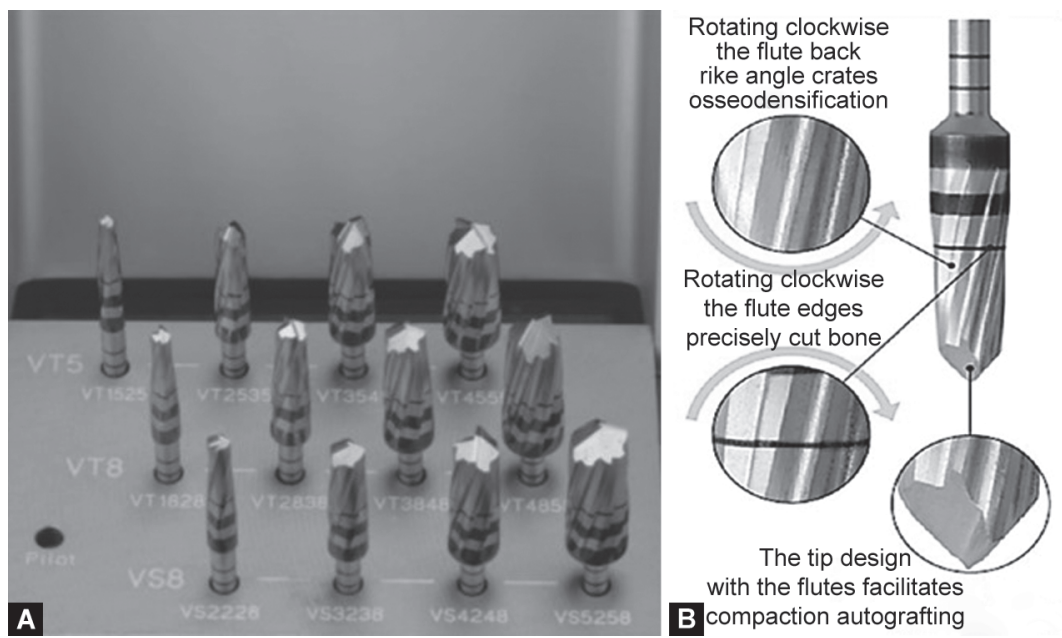

Figs $1 \mathrm{~A}$ and $\mathrm{B}$ : (A) Densah ${ }^{\mathrm{TM}}$ Bur used for the study; (B) Osseodensification cutting concept
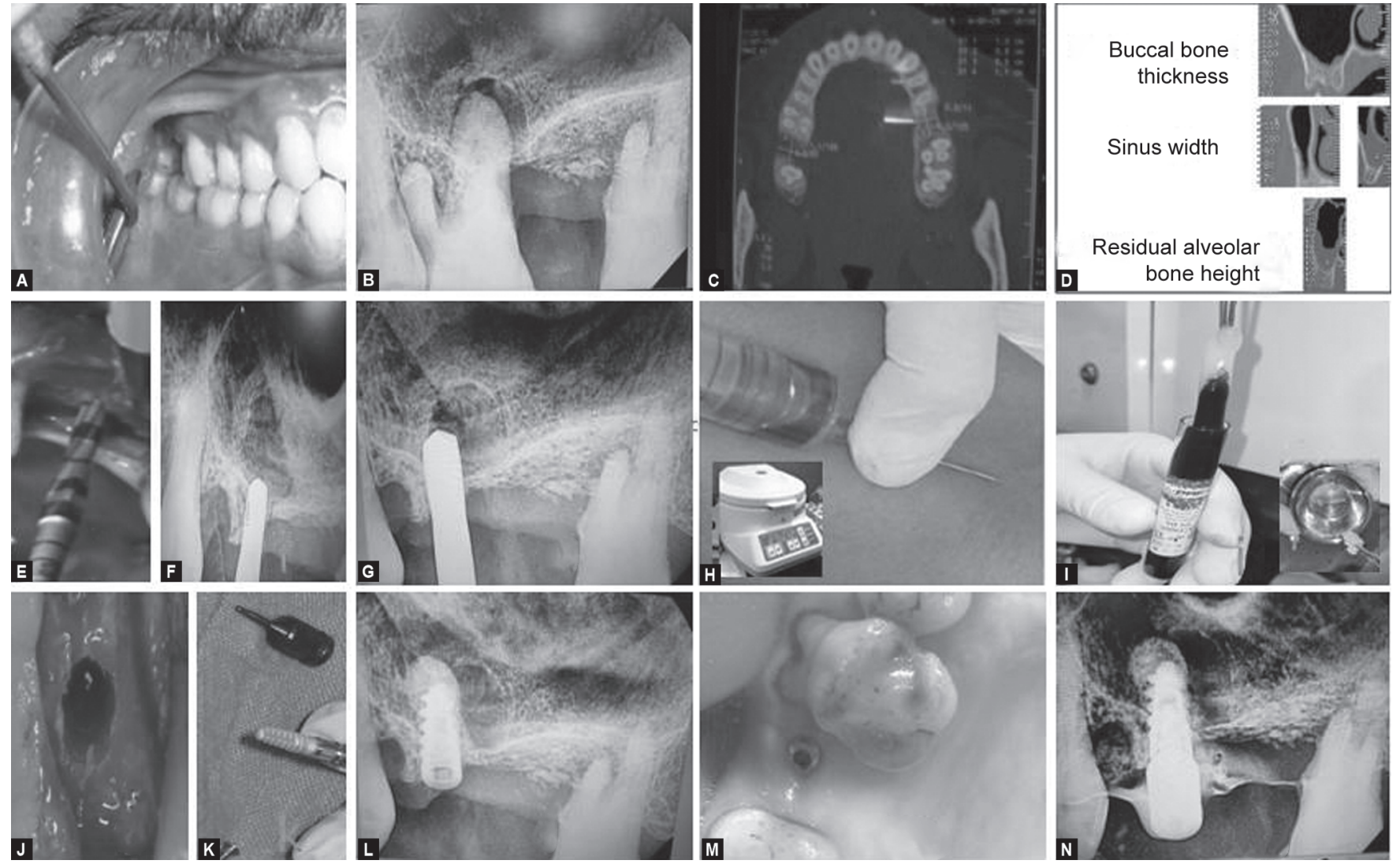

Figs 2 A to N: (A) Preoperative; (B) Preoperative X-ray; (C) Preoperative CT scan; (D) Residual bone height; (E) First Densah ${ }^{\text {TM }}$ Bur; (F) X-ray-first Bur position; (G) X-ray-sequential bur in densifying mode; (H) PRF and centrifuge protocol; (I) Sticky bone prepared; (J) Final width osteotomy; (K) Implant planned; (L) Implant inserted; (M) Postoperative; (N) Healing abutment for prosthesis

confirmed on the radiograph. As per the manufacturer's instructions, sequential widening (Fig. 2G) was done changing the drill motor to reverse-densifying mode with a gentle pumping motion. Additional vertical depth and membrane lift (in $1.0 \mathrm{~mm}$ increments) were achieved in the process. The stratagem was performed throughout with an intermittent pressure of 1 second on the bone and 1-2 seconds off the bone under copious saline irrigation.

Next, the patients' blood was drawn from the median cubital vein in a test tube and centrifuged in a Process ${ }^{\mathrm{TM}}$, France assemblage emulating a standard protocol (Fig. 2H). The spin-off was perceived as three layers; plasma on top, PRF in the middle, and red corpuscle layer at the bottom. The procured clot was mixed with the graft NB Dental Morsels, NovaBone ${ }^{\circledast}$ (Fig. 2l) to form a sticky bone. As planned (Fig. 2J), the $3.75 \times 10 \mathrm{~mm}$ implant, removed from the sterile vial (Fig. 2K) drove the biomaterial under $35 \mathrm{rpm}$ (Fig. 2L) into the final desired depth. Upon ensuring a $25-\mathrm{Ncm}$ torque stability (Fig. $2 \mathrm{M}$ ), a healing abutment was tightened with a hex driver (Fig. 2N) over it and the site was sutured finally. Postoperative prescription 


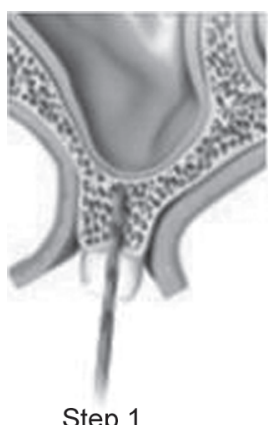

Step 1

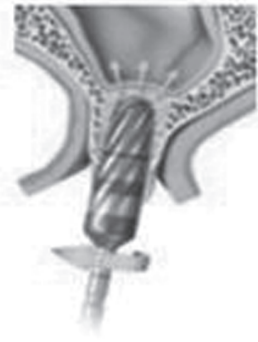

Step 2

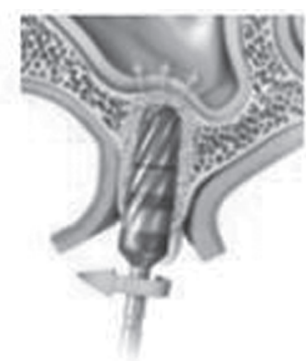

Step 3

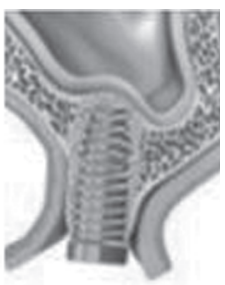

Step 4

Fig. 3: Implant placement and sinus lift by densification

for accelerated healing included mouthwashing, analgesics, Blue ${ }^{\circledR} \mathrm{m}$ oral gel application thrice daily, and antibiotics for a week.

\section{Discussion}

Elevation of the maxillary sinus floor was first reported by Boyne in 1960. Various intracrestal SFE techniques were proposed thereafter including bone condensation by the use of osteotomes (summers-and its multiple variations), balloon lift, and hydraulic sinus condensing technique (Chen and Cha). But at sinus proximity, the associated risks of paresthesia, perforation, and patient morbidity cannot be ignored. Moreover, Summer's method resulted in microfractures in the peri-implant bone, thus jeopardizing osseointegration (OI). Besides, an implant diagnosed as a clinical failure is easier to describe than one that is a success. ${ }^{5}$ The negative effects of parafunctional forces and smoking on the early loss of rigid fixation have been established. ${ }^{9}$ Therefore, the threats risking failure were taken into account for case selection. The trials on short implants with improvised surface geometries ${ }^{9}$ have presented to bypass the SFE necessitation and are predictable alternatives, as well.

With the growth factor-dominated regeneration looming and PRF having neo-antigenic characteristics, ${ }^{10}$ it was used with an osteostimulant for our patient. The sticky bone was used with similar manipulation in the study by Soni et al. for the dental implants. ${ }^{11}$ Formation of a direct bone-to-implant interface $(\mathrm{BIC})$ is a consistent treatment goal in implant dentistry. ${ }^{12}$ Implant stability is pivotal for $\mathrm{Ol}$ and the determining factors are bone density, surgical protocol, and implant thread type, and geometry. ${ }^{3,5}$ The SD for the osteotomy process works by cutting the bone for the implant's geometry. Circumstantially, the implant insertion torque is reduced leading to poor primary stability and potential lack of integration. ${ }^{7}$

On the contrary, the OD technology uses multi-grooved burs in the counterclockwise direction (Figs $1 \mathrm{~A}$ and $\mathrm{B}$ ) that favorably undersized the implant bed preparation. It is hypothesized that bone will be pushed toward the apical end and will begin to gently lift the Schneiderian membrane. The outward autografting is done in four steps (Fig. 3) and the same was evident on radiographs recorded at appropriate stages (Figs 2F, G, L, and N). Nonetheless, an increase in BIC and the bone area fraction occupancy (BAF) was observed, as a secondary finding in a very recent systemic review. ${ }^{13}$ However, more well-designed randomized controlled trials are required for the biologic plausibility.

\section{CONCLUSION}

The explicated methodology is feasible in the dental clinical setup for its ease and non-invasiveness.

\section{Clinical Significance}

Sinus implantology is challenged with ever-increasing demand for permanent prosthetic rehabilitation in jaw bone sites with marked limitations.

\section{References}

1. Misch CE. Rationale for dental implants. In: Misch CE, ed. Contemporary implant dentistry. St. Louis: Mosby; 1999. pp. 3-12.

2. Mendonca G, Mendonca GB, Fernandes-Neto AJ, et al. Management of fractured dental implants; a case report. Implant Dent 2009;18(1):1016. DOI: 10.1097/ID.0b013e318192cafe.

3. Misch CE. Density of bone: effect on treatment plans, surgical approach, healing, and progressive loading. Int J Oral Implant 1990;6(2):23-31.

4. Ulm CW, Solar P, Krennmair G, et al. Incidence and suggested surgical management of septa in sinus-lift procedures. Int J Oral Maxillofac Implants 1995;10(4):462-465.

5. Munjal S, Munjal S, Hazari P, et al. Evaluation of specifi cally designed implants placed in the low-density jaw bones: a clinicoradiographical study. Contemp Clin Dent 2015;6(1):40-43. DOI: 10.4103/0976-237X.149290.

6. Tan WC, Lang NP, Zwahlen M, et al. A systematic review of the success of sinus floor elevation and survival of implants inserted in combination with sinus floor elevation. Part II: transalveolar technique. J Clin Periodontol 2008;35(Suppl):241-254. DOI: 10.1111/j.1600-051X.2008.01273.x.

7. Huwais S, Meyer EG. A novel osseous densification approach in implant osteotomy preparation to increase biomechanical primary stability, bone mineral density, and bone-to-implant contact. Int J Oral Maxillofac Implant 2017;32(1):27-36. DOI: 10.11607/jomi.4817.

8. Trisi P, Perfetti G, Baldoni E, et al. Implant micromotion is related to peak insertion torque and bone density. Clin Oral Implants Res 2009;20(5):467-471. DOI: 10.1111/j.1600-0501.2008.01679.x.

9. Munjal S, Munjal S. Short $(5.0 \times 5.0 \mathrm{~mm})$ dental implant placement: a case report with 3 year follow up. Mod App Dent Oral Health 2019;4(1):316-319.

10. Dohan DM, Choukroun J, Diss A, et al. Platelet-rich fibrin (PRF): a second-generation platelet concentrate. Part I: technological concepts and evolution. Oral Surg Oral Med Oral Pathol Oral Radiol 2006;101(3):37-44. DOI: 10.1016/j.tripleo.2005.07.008.

11. Soni R, Priya A, Yadav H, et al. Bone augmentation with sticky bone and platelet-rich fibrin by ridge-split technique and nasal floor engagement for immediate loading of dental implant after extracting impacted canine. Natl J Maxillofac Surg 2019;10(1):98-101. DOI: 10.4103/njms.NJMS_37_18.

12. Munjal S, Munjal S. Immediate implant loading: current concepts: a case report. J Orofac Res 2011;1:34-38.

13. Padhye NM, Padhye AM, Bhatavadekar NB. Osseodensification -- a systematic review and qualitative analysis of published literature. J Oral Biol Craniofac Res 2020;10(1):375-380. DOI: 10.1016/j. jobcr.2019.10.002. 